\title{
Gestión tecnológica para PYMES: Aspectos legales y financiación
}

\author{
Technological management for SMEs: Legal aspects and financing
}

\author{
Guillermo Hernán Morales Mancilla, Rita Mabel Villegas \\ g.moralesmancilla@gmail.com,rimavillegas@yahoo.com.ar \\ Universidad nacional de la Patagonia Austral, Unidad Académica Rio Gallegos, Instituto de \\ Trabajo, Economía y Territorio (I.T.E.T.) \\ Av. Gobernador Gregores y Piloto "Lero" Rivera. Rio Gallegos, Santa Cruz, Argentina.
}

Recibido: 04/05/2020. Aceptado: 17/11/2020

\section{RESUMEN}

En el presente informe se investigó mediante una metodología explorativa descriptiva, sobre el marco legal y las oportunidades de financiamiento en cuestiones de gestión tecnológica para las Pymes de la localidad. Resultó de suma importancia conocer la existencia de legislación respecto a la gestión tecnológica y se procedió a compendiar qué reglamentan las leyes nacionales $\mathrm{N}^{\mathrm{o}} 23.877$ y N $\mathrm{N}^{\mathrm{o}} 25.467$. Por otro lado, a través de las paginas oficiales de las entidades encargadas de lanzar las convocatorias de las líneas de créditos vigente en los años 2015 - 2018, se descubrió la existencia de varias líneas de financiamiento e incentivos y se logró exponer las principales líneas que fueron ofrecidas por el estado tanto nacional como provincial, las cuales son muy variadas y se ajustan de manera directa o indirecta a la gestión tecnológica de las Pymes.

Palabras clave: Tecnológica; Pymes; Financiamiento; investigación; Innovación.

\begin{abstract}
In this report, a descriptive exploratory methodology was investigated on the legal framework and financing opportunities in technology management issues for local SMEs. It was very important to know the existence of legislation regarding technology management and we proceeded to compile what national laws $\mathrm{N}^{\circ} .23,877$ and $\mathrm{N}^{\circ} .25,467$ regulate. On the other hand, through the official pages of the entities in charge of launching the calls for the credit lines in force in the years 2015 - 2018, the existence of several financing and incentive lines was discovered and it was possible to expose the main lines that were offered by the state, both national and provincial, which are very varied and adjust directly or indirectly to the technological management of SMEs.
\end{abstract}

Keywords: Technology; SMEs; Financing; research; Innovation.

\section{INTRODUCCIÓN}

El presente informe es realizado en el marco del proyecto de investigación: 29/A422 "Gestión de innovación tecnológica y creatividad en las Pymes de Rio Gallegos - Santa Cruz" dirigido por la Mg. Rita Mabel Villegas. Fue designado investigar sobre el marco legal y las 
oportunidades de financiamiento en cuestiones de gestión tecnológica para las Pymes de la localidad.

En estos tiempos modernos el ambiente económico es sumamente complejo para las Pymes que interactúan en el mismo a nivel nacional, provincial y al que no escapa tampoco nuestra ciudad Rio Gallegos, sumando a esto que el sector privado es pequeño comparado con el sector estatal, brinda un panorama pesimista. A pesar de esto se ha observado que existen empresarios que apuestan a la gestión tecnológica, como una posible solución para combatir dicho ambiente complejo, mejorando a partir de ésta su competitividad; ajustando tecnología, procesos y talentos humanos de cara al contacto con el entorno generando una cultura proactiva y de cambio.

Dada esta realidad es importante conocer si existen y cuáles fueron las políticas de financiamiento e incentivos adoptados en el periodo 2015-2018 por el Estado Nacional, Provincial o Municipal hacia los empresarios locales que permiten mejorar la posición competitiva de las Pymes a través de la gestión tecnológica o de la adecuación, desarrollo y adquisición de nuevas tecnologías relevantes para el futuro de las mismas. Para ello es necesario detectar si la gestión tecnológica forma parte de una cultura empresarial o si la misma es posible por una política de apalancamiento financiero u operativo que desarrolla el Estado.

Por lo expuesto es necesario indagar sobre: ¿Existen políticas de financiamiento para la gestión tecnológica? ¿Cuáles con las líneas de apalancamiento financiero u operativo que permiten desarrollar en las Pymes gestión tecnológica con recursos del Estado? ¿Las líneas de financiamiento contribuyen a desarrollar una cultura de la gestión tecnológica en las Pymes? La investigación bajo estos aspectos posibilitará aproximar el análisis y caracterización de la legislación vigente entre 2015-2018 y conocer la existencia de una cultura de cambio en la organización interna de la Pyme que posibilite un mejor posicionamiento y transformación del entorno que la rodea. Asimismo, se proporcionará una comprensión sobre el estado actual de la relación entre el Estado y las Pymes locales en materia de promoción, apoyo e incentivo para el crecimiento, desarrollo y sustentabilidad de las mismas.

La información obtenida para la conformación del informe fue consultada de páginas oficiales del Ministerio de Ciencia, Tecnología e Innovación de Argentina, Consejo Federal de Inversiones (CFI), Ministerio de Producción de la provincia de Santa Cruz, InfoLEG. Además de trabajos científicos de investigadores de envergadura internacional y bibliografía especifica en materia de innovación y gestión tecnológica.

Marco histórico:

\section{MARCO DE REFERENCIA}

Como resultado de una exploración de informes técnicos relacionados a los temas estudiados por el proyecto de investigación, se han obtenido algunos casos relacionados al tema particular que se desarrolla en el presente informe científico técnico.

Existen antecedentes en Colombia en el año 2017, en el cual se investigó el tema que se desarrolla en el presente informe, el trabajo científico de tres autores denominado "Direccionamiento Estratégico: Proyectos de la innovación Tecnológica y Gestión Administrativa en las Pequeñas Empresas", expone que las políticas estatales son de suma importancia para apoyar a las pequeñas y medianas empresas que generan el $67 \%$ del empleo en el país, de este análisis ha surgido la necesidad de analizar el comportamiento de estas empresas, en cuanto a temas como la supervivencia en el mercado y los motivos que contribuyen al éxito o fracaso de su permanencia en el tiempo, es ahí donde surge la gestión tecnológica como un factor determinante. Y en los resultados de las entrevistas a empresas del sector privado que prestan servicios de financiamiento a las Pymes de Colombia, éstas prevén en un corto plazo, que se presentará un aumento considerable en productos dirigidos a esta 
clase de empresa, por lo que se concluye como una deficiencia de las políticas públicas de incentivo y apoyo a la gestión tecnológica para las pymes de ese país.

También otro informe de Colombia pero del año 2015, denominado "Estrategias de innovación y capital social en la pequeña y mediana empresa", el cual sostiene que la globalización exige trascender las barreras de competitividad para redimensionar y alcanzar el desarrollo nacional. También expresa que es el estado el encargado, mediante políticas públicas, de promover la competitividad, ya que viene impulsando procesos que a nivel sistémico apoya estos rumbos. Luego del proceso de investigación como resultado para evaluar aspectos de competitividad se obtuvo que se deben combinar la innovación tecnológica y el capital social como factores clave de éxito.

Marco Conceptual:

La Organización para la Cooperación y Desarrollo Económico (OCDE), definió en 1981 la innovación como "todos los pasos científicos, comerciales, técnicos y financieros necesarios para el desarrollo e introducción en el mercado con éxito de nuevos o mejorados productos, el uso comercial de nuevos o mejorados procesos y equipos, o la introducción de una nueva aproximación a un servicio social. La I+D es sólo uno de estos pasos".

También ésta organización detalla los tipos de innovación que existe, estos son: innovación en productos o servicios, innovación en procedimientos y procesos, innovación en comercialización y por ultimo innovación en organización.

Luego en 1996 se definió la innovación tecnológica como la aplicación de la ciencia y la tecnología en una nueva dirección, seguida de un éxito comercial, en un mercado o aparato económico donde la competencia es vital.

En el mundo de las Pymes, la innovación tecnológica, surge cómo una estrategia en respuesta a los desafíos impuestos por la globalización de la economía, por los avances tecnológicos y la expansión del uso de nuevas herramientas en información y comunicaciones. Cuando se habla de innovación tecnológica en el mundo empresarial, hay que hacer referencia a las mejoras en productos o servicios que ya existen en el mercado, introducir productos que ofrezcan algún plus o valor agregado, actualizar los sistemas de información, renovar herramientas tecnológicas o maquinarías y equipos que permitan aumentar la productividad en las empresas, la gestión de cambios en organización y administración de las empresas (Riascos et al., 2016). ${ }^{1}$

Marco Teórico:

Se observa que la mayor parte de las Pymes de Rio Gallegos son caracterizadas como altamente "rutinarias", así sucede también en gran parte de Argentina, si se considera la representación en la matriz de Jorge Hermidas cuando plantea en su libro "Administración y Estrategia" cuatro modelos culturales que posibilitan poseer un razonamiento y diagnostico asociado a la alta visión creativa y acción empresaria.

Sin embargo, existe una porción del empresariado local que está atento a los cambios de métodos, sistemas y procedimientos en cuanto hace a la tecnología e innovación para su supervivencia en el mercado que opera. Juan Carlos Fresco señala en su libro "El proceso de transformación y cambio en las organizaciones" al referirse al ciclo de vida de la empresa y la mentalidad del empresario que: "En la etapa de desarrollo es primordial la existencia de una cultura ganadora que capte tecnología, la incorpore, busque y determine el o los segmentos de mercado al cual o a los cuales apuntará. La investigación es el aliado que provee el conocimiento para encausar la acción hacia el "target" más conveniente" (2005: Pág.: 13).

El marco teórico que se ha seleccionado tal como lo señala Fresco (Pág. 18-19) se asociará a "la mentalidad de la nueva ola que vivimos, necesaria para afrontar la competitividad y la realidad de estos tiempos y que nos abre la puerta de este Siglo XXI que, sin duda, tal como

\footnotetext{
${ }^{1}$ Cardona Diego, Del Rio Jorge y Hernández Hugo (2017) "Direccionamiento Estratégico: Proyección de la Innovación Tecnológica y Gestión Administrativa en las Pequeñas Empresas”. Colombia. Página 2
} 
lo vaticinó Toffler, abrirá una creciente distancia entre el mundo rápido y el mundo lento.” La creación de riqueza entre las sociedades en la actualidad "se encuentra impulsadas por el conocimiento y la tecnología avanzada y las sociedades que se quedaron empantanadas en la "agricultura tradicional" o en "industrias de chimeneas con burocracia", constituyen el gran desafío" del siglo." El modelo burocrático no puede adaptarse a la velocidad de cambio existente en el mundo actual y son devastados por aquellos que en los negocios son veloces cazadores, que estudian el terreno, hábitos, costumbres, adelanto tecnológico, invierten en investigación y desarrollo, e incorporan recursos humanos competitivos. El marco teórico expuesto se relaciona con el estado actual del conocimiento a partir de Bibliografía seleccionada y en las investigaciones anteriores realizadas.

Marco legal:

Este marco es de suma importancia para abordar el presente informe, debido a que es uno de los objetivos del mismo conocer si existe legislación con respecto a la gestión tecnológica y de ser así, referenciar que reglamentan dichas leyes. Existe legislación vigente a nivel nacional con respecto al tema ya mencionado, las leyes que entran en vigor en este caso son las leyes $\mathrm{N}^{\mathrm{o}} 23.877$ y 25.467 .

En el caso de la ley 23.877 "Promoción y fomento de la innovación tecnológica", tiene como objeto mejorar la actividad productiva y comercial, a través de la promoción y fomento de la investigación y desarrollo, la transmisión de tecnología. Los beneficiarios son personas físicas y jurídicas, públicas o privadas que desarrollen actividades productivas, científicas, tecnológicas o financieras.

Las iniciativas para la promoción y fomento de la innovación se llevará a cabo mediante distintos mecanismos, estos son: De promoción y fomentos financieros (a cargo de entidades financieras, habilitadas para tales efectos por el BCRA), De promoción y fomento fiscales (El Poder ejecutivo nacional fijara anualmente un cupo de créditos fiscales, que podrá ser utilizado para la modalidad que especifica la ley), De promoción y fomento no financiero (Serán provistas por el Estado, de acuerdo a previsiones presupuestarias, aportes del tesoro o que sean adjudicadas con cargo de devolución pero sin intereses) y De promoción y fomento especiales (los que fueran creados transitoria o permanentemente y que no están contemplados en otra categoría).

En su artículo 11, inciso a) establece a la Micro, pequeña y mediana empresa como prioridad para el objeto de la presente ley. Y será la secretaria de Ciencia y Tecnología de la Nación la autoridad de aplicación de la ley, cumpliendo todas las funciones que se detallan en el cuerpo de la misma. La forma de distribución es el 25\% para la nación, y el $75 \%$ restante para las provincias, donde destaca Buenos Aires con 17\% como el mayor monto porcentual de todas las provincias. Santa Cruz recibe solo el $2.5 \%$ del total, entrando en el grupo de provincias que recibe la menor cantidad del fondo.

Con respecto a la ley 25.467 "Ciencia, Tecnología e Innovación”, tiene como objeto establecer un marco general que estructure, impulse y promueva las actividades de ciencia, tecnología e innovación, proponiendo el bien común, al fortalecimiento de la identidad nacional, a la generación de los trabajos y a la sustentabilidad del medio ambiente.

Con respecto a las responsabilidades indelegables del Estado nacional en materia de política científica, tecnológica y de innovación, se destaca a lo que hace al estudio particular del informe, el inciso b) del artículo 5 de la presente ley, el cual dicta financiar una parte substantiva de la actividad de creación de conocimiento conforme con criterios de excelencia. También formula las políticas y establece mecanismos, instrumentos e incentivos necesarios para que el sector privado contribuya a las actividades e inversiones en el campo científico, tecnológico e innovativo.

Por último, el capítulo 5 expone el financiamiento de las actividades de investigación y desarrollo. Concurren al financiamiento del sistema nacional de ciencia, tecnología e 
innovación: el estado nacional mediante partidas presupuestarias asignadas correspondiente a la función de Ciencia y tecnología, las provincias y CABA, a quienes se los invita a establecer niveles presupuestarios similares al estado nacional. Las empresas privadas, instituciones $\mathrm{u}$ organismos no gubernamentales que realicen promoción y ejecución de estas actividades y aporte públicos o privados externos.

Materiales y métodos:

\section{RESULTADOS, ANÁLISIS Y DISCUSIÓN}

Para este proyecto se ha definido que la metodología de investigación sea de tipo explorativa descriptiva. Se propuso a través de la recopilación de datos de las paginas oficiales de las siguientes instituciones a fin de realizar el trabajo de campo: Instituto Nacional de Tecnología Agropecuaria, Consejo Federal de Inversiones, Ministerio de Ciencia, Tecnología e Innovación de Argentina, Ministerio de Producción de la Provincia de Santa Cruz (Dirección Provincial de Proyectos y de Tecnología) y Dirección Desarrollo Productivo local del Municipio de Río Gallegos, para conocer cómo fue la dinámica de apoyo por parte del Estado hacia los empresarios pymes locales y obtener una primera aproximación para observar por un lado las líneas que estaban vigentes de apoyo a las Pymes y por el otro obtener información de cuáles son las empresas que han sido beneficiadas en el periodo 2015/2018 por las actuales políticas de incentivos hacia la tecnología.

La investigación bajo estos aspectos proporcionó una comprensión sobre la relación entre el Estado y las Pymes locales en materia de promoción, apoyo e incentivo para el crecimiento, desarrollo y sustentabilidad de las mismas. A medida que se avanzó con el proceso de investigación se prepararon los respectivos Abstrac, la metodología del análisis y selección de datos y preparación de informes. Las fuentes secundarias que fueron utilizadas fue bibliografía adecuada que apoya el marco teórico, la obtención de información elaborada por las instituciones y legislación vigente e informes científicos de otros autores, incluidos los que elaboró parte del equipo de investigación anteriormente, entre otras que puedan obtenerse. Una vez terminada la etapa exploratoria, se procedió a trabajar sobre las conclusiones arribadas en el análisis y caracterización de la legislación actual sobre la cultura de la gestión tecnológica en las Pymes de Rio Gallegos. (2015-2018) La transferencia de los resultados obtenidos será realizada hacia diferentes actores internos y externos a la Universidad lo que será la culminación del presente trabajo.

Resultados:

Como ya se mencionó con anterioridad conocer la existencia de la legislación vigente en el ámbito Nacional, Provincial y Municipal para el desarrollo de la gestión tecnológica e innovación facilita a que las Pymes sean competitivas aún en escenarios complejos y con entornos cambiantes. El acceso a la información por parte del Pyme sobre los incentivos y posibilidades de apalancamiento (operativo o financiero) facilitan una cultura de gestión tecnológica. Los cambios que se producen en el medio externo son relevantes y pueden afectar los productos, servicios, mercados, proveedores, distribuidores, competidores, clientes, procesos productivos, procesos de comercialización e inclusive puede afectar la posición del mercado de la Pyme.

Es necesario entonces que las empresas puedan planificar la gestión tecnológica ya que ésta puede operar creando nuevas oportunidades o bien transformando el contexto. Al poseer mayor flexibilidad y capacidad de reacción que la gran empresa por su tamaño y estructura, la Pyme puede adaptarse con mayor facilidad. Sin embargo, uno de los aspectos que la pueden perturbar es el ámbito financiero frente a la escaza disponibilidad de contar con el capital propio o a veces la dificultad para acceder a líneas de financiación que les permita adaptarse a ellas. 
Luego de la labor explorativa mencionada en el apartado precedente, se obtuvo la siguiente información con respecto a las líneas de financiamiento (2015-2018), con respecto al orden nacional las principales líneas fueron presentadas por entidades estatales como el Ministerio de ciencia, tecnología e innovación de Argentina (MinCyT), la confederación Argentina de Media Empresa (CAME) y el Ministerio de desarrollo de la nación.

La Agencia Nacional de Promoción Científica y Tecnológica, a través del Fondo Tecnológico Argentino (FONTAR) se generaron varias líneas de financiamiento, promoción y/o incentivos para las pymes. Una de estas líneas "FIN SET 2015", en dicho año se llamó a la presentación de proyectos para fortalecer y desarrollar capacidades para la prestación de servicios tecnológicos orientados a la producción de bienes y servicios, apoyando la ampliación o modernización de infraestructura y talentos en carácter de aporte no reembolsable (ANR). El monto de financiamiento podía ser de 4.5 millones hasta 14.5 millones de pesos, y no podía ser superado el $80 \%$ del costo total del proyecto y con un plazo de construcción no mayor a 18 meses contados desde los 90 días posteriores a la firma del contrato.

También mediante la misma Agencia se presentó el ANR ASIS-TECi, para financiar proyectos de asistencia tecnológica, ayudando a las empresas a identificar y solucionar retos tecnológicos y estimular el desarrollo tecnológico de forma permanente mejorando las capacidades de innovación. El mismo consistió en una subvención no reintegrable de hasta 225.000 pesos por empresa y no conseguía superar el $60 \%$ del costo total del proyecto y el plazo máximo para ser ejecutado era de 12 meses.

El ANR Social, "Aportes No Reembolsable para Desarrollos Tecnológicos con Impacto Social", tuvo convocatoria en el año 2016 y también en el año siguiente. El objetivo fue financiar parcialmente proyectos que tengan como meta la innovación tecnológica para impulsar el desarrollo social y territorial, con eje en poblaciones vulnerables, pequeños productores y micro emprendimientos. Se pensó bajo dos modalidades con fines distintos, en el primer caso orientado a fortalecer capacidades tecnológicas de un sector productivo de la economía social y en el segundo caso orientado al usuario final. En ambos casos los periodos máximos de ejecución fueron de 24 meses y con un tope del $80 \%$ del costo total del proyecto. Los montos máximos fueron para el 2016 modalidad 1 hasta 3.5 millones de pesos y en el caso de la modalidad 2 hasta 2.25 millones. En el caso del año 2017 para ambas modalidades el monto máximo de la subvención fue de 4.8 millones de pesos.

Por ultimo a lo que refiere al Ministerio de Ciencia, Tecnología e Innovación, se desplegó el "Aporte No Reembolsable Desarrollo Tecnológico" (ANR PDT), el cual tuvo invitación en 3 años consecutivos, bajo las denominaciones "ANR 2200” 2015, “ANR 3500” 2016 y "ANR 4800" 2017. En todos los casos el objetivo fue el mismo, financiar parcialmente proyectos que tengan como meta mejorar las estructuras productivas y la capacidad innovadora de las empresas de distintas ramas de actividad, mediante "proyectos de innovación y desarrollo tecnológico", que conduzcan a generar innovaciones a nivel nación de productos y/o procesos. También sobrellevaron el mismo límite de plazo de ejecución de 36 meses y el aporte no podía superar el $80 \%$ del valor del proyecto, de esta manera lo único que se modifico fue el importe máximo; en 2015 fue de 2.2 millones de pesos; en 2016 fue 3.5 millones de pesos y por último en 2017 fue de 4.8 millones de pesos.

Por otro lado en la Red CAME se presentó en el año 2017, a través de su departamento de programas de financiamiento y competitividad Pyme, el programa FONARSEC EMPRETECNO (financiamiento para emprendedores tecnológicos), que fue implementado por la Agencia Nacional de Promoción Científica y Tecnológica del Ministerio de Ciencia, Tecnología e Innovación Productiva de la Nación. Se destinó a desarrollar emprendimientos de intensidad tecnológica y con fines de crear nuevas empresas de base tecnológica, tanto para instituciones públicas como privadas dedicadas a actividades de desarrollo científicotecnológicas y de innovación. El financiamiento de los proyectos fue de entre 1.5 millones de 
pesos hasta 6 millones, siempre que no supere el $80 \%$ del costo del proyecto, con un plazo de 12 meses.

Desde el mismo departamento de esta entidad pero en el año 2018 surgió el Programa Fondo Semilla, con un financiamiento a modalidad de préstamo de hasta $\$ 250.000$ a tasa $0 \%$. Para acceder se debía tratar de un emprendimiento nuevo, o la vida del mismo no debía superar los 12 meses, debidamente inscripto en AFIP, para proyectos productivos y/o de desarrollo tecnológico, y en el caso del sector servicios proyectos realmente innovadores.

Y por último desde el Ministerio de Desarrollo se impulsó en 2017 una herramienta PAC EMPRESA, que buscó contribuir al aumento de la competitividad en las Pymes, con un préstamo de hasta $\$ 400.000$ como aporte no reembolsable (sin devolución), siempre que la pyme acredite 2 años de actividad económica verificable y que el monto no supere el $60 \%$ del proyecto, si bien la convocatoria fue abierta, al tener como fin aumentar la competitividad, es de utilidad mencionarla en el informe.

En lo que hace referencia al orden Provincial, el Ministerio de Producción, Comercio e Industria, a través de la secretaria de Comercio e Industria, se lanzó oficialmente "Santa Cruz Produce", programa que brindaba distintas líneas de financiamiento para emprendedores, pequeños y medianos empresarios e incluso comerciantes que cumplan con un plazo mínimo de actividad de 2 años. La convocatoria es abierta para todos los proyectos, pero a través de una comunicación vía E-mail con la Dirección Provincial de Proyectos del Ministerio de la Producción de la Provincia de Santa Cruz, se obtuvo la información que los proyectos innovadores son prioridad para los créditos PRODUCE.

Las líneas ofrecidas fueron: "JOVEN EMPRENDEDOR", el cual contó con un monto de hasta $\$ 300.000$, destinado a personas con título secundario de 18 hasta 30 años de edad, el plazo fue de 48 meses como máximo y un periodo de gracia de hasta 18 meses. La financiación es hasta el $100 \%$ del proyecto a una tasa fija del $8 \%$ y no se requirió garantía. Por otro lado el "CREDITO AVANZAR" que poseía un monto de $\$ 500.000$ y como destinatario micro empresas, también una duración máxima de 48 meses y un plazo de gracia en este caso de 12 meses. Financió el 100\% del proyecto, a una tasa del 19\% y con una garantía o hipoteca del $100 \%$.

Y por último la línea "CREDITO FORTALECER", la cual fue la de mayor monto, $\$ 1.000 .000$ por lo cual requiere mayores requisitos, fue destinada a Pymes, con un plazo máximo de hasta 84 meses y el plazo de gracia de 24 meses. Financiaba hasta el $80 \%$ del proyecto a una tasa fija del $21 \%$ y con una prenda o hipoteca del $130 \%$.

\section{CONCLUSIONES}

Posteriormente al desarrollo del Informe Científico Técnico se arribó a las siguientes conclusiones:

Para dar respuesta a las preguntas elaboradas en la introducción, se confirma que existe legislación a nivel nacional que compromete al Estado a financiar proyectos y actividades de innovación y gestión tecnológica, a través de la Ley Nacional No 23.877.

También se demostró que existen líneas de financiamiento e incentivos y se pudieron exponer las principales líneas de apalancamiento que ofrece el estado tanto nacional como provincial, las cuales son muy variadas y de diferentes entidades, que se ajustan de manera directa o indirecta a la gestión tecnológica de las Pymes.

Con respecto al último interrogante, no se pudo dar respuesta debido a que no se tuvo acceso a entrevistas con empresarios Pymes, como para obtener un Feedback e interpretar si el financiamiento desarrolla una cultura de innovación tecnológica. Lo cual se destaca ya que queda pendiente para una futura investigación. 


\section{RECOMENDACIONES}

Las recomendaciones que se puede aportar después de la investigación realizada es hacia las dos partes que hemos trabajado en el presente informe.

Primeramente al Estado, en todos sus niveles, se recomienda trabajar en políticas públicas de marketing, publicidad y promoción de las líneas que ofrece para llegar a un público objetivo mayor y así poder potenciar el crecimiento de las Pymes que va a repercutir como un crecimiento de la economía local, provincial y nacional.

Luego también invitar a que los empresarios modernos tomen estas financiaciones como una gran oportunidad para su proyecto, emprendimiento o empresa y sumar a lo que se nombró en el marco teórico como empresarios rutinarios a que rompan el paradigma y también incursionen en este mundo moderno para obtener ventajas y no quedar "fuera del juego".

\section{AGRADECIMIENTOS}

A la directora del Proyecto de investigación Mg. Rita Villegas, por la oportunidad para formar parte del equipo de investigación, el cual fue un aporte muy significativo a mi formación personal y profesional.

A Mg. Mónica Manuelides, Lic. Sandra Ortiz, Ing. Allan Beecher e Ing. Mauro Ziehlke, miembros del equipo de investigación multidisciplinario por sus aportes a la formación científica.

A los profesores Jorge Lescano y Luis Sierpe por sus correspondientes recomendaciones para merituar en la beca de investigación.

Al Sr. Daniel Ríos y todo el equipo de la Secretaria de Investigación y Posgrado por el asesoramiento y seguimiento continuo para transitar de una manera más amena el nuevo desafío de iniciar en la investigación.

A la Profesora Fabiola Mancilla por trasmitir de manera desinteresada conocimientos a lo que refiere a la escritura científica y redacción para la confección del presente informe.

\section{REFERENCIAS}

AGENCIA NACIONAL DE PROMOCIÓN CIENTÍFICA Y TECNOLÓGICA 01 de Septiembre del 2015. ANR 22002015 C3". Link: http://www.agencia.mincyt.gob.ar/ frontend/agencia/convocatoria/347

AGENCIA NACIONAL DE PROMOCIÓN CIENTÍFICA Y TECNOLÓGICA 08 de Septiembre del 2015. FIN SET 2015". Link: http://www.agencia.mincyt.gob.ar/ frontend/agencia/convocatoria/349

AGENCIA NACIONAL DE PROMOCIÓN CIENTÍFICA Y TECNOLÓGICA 12 de Abril del 2016. ASIS - TECi”. Link: http://www.agencia.mincyt.gob.ar/frontend/ agencia/convocatoria/364

AGENCIA NACIONAL DE PROMOCIÓN CIENTÍFICA Y TECNOLÓGICA 23 de Agosto del 2016. ANR 35002016 C3". Link: http://www.agencia.mincyt.gob.ar/frontend/ agencia/convocatoria/381

AGENCIA NACIONAL DE PROMOCIÓN CIENTÍFICA Y TECNOLÓGICA 23 de Agosto del 2016. ANR SOCIAL 2016 C2”. Link: http://www.agencia.mincyt.gob.ar/frontend/ agencia/convocatoria/380

AGENCIA NACIONAL DE PROMOCIÓN CIENTÍFICA Y TECNOLÓGICA 20 de Marzo del 2017. ANR 48002017 C1". Link: http://www.agencia.mincyt.gob.ar/frontend/ agencia/convocatoria/392 
AGENCIA NACIONAL DE PROMOCIÓN CIENTÍFICA Y TECNOLÓGICA 14 de Agosto del 2017. ANR SOCIAL 2017 C2". Link: http://www.agencia.mincyt.gob.ar/frontend/ agencia/convocatoria/405

BRAVO M., MENJÍA A. y MENDIETA C. (2015). "Estrategias de innovación y capital social en la pequeña y mediana empresa". Colombia Recuperado el 25 de abril de 2020 de http://scielo.sld.cu/scielo.php?pid=S1815-59362015000300006\&script=sci abstract

CARDONA D., DEL RIO J. y HERNÁNDEZ H. (2017) “Direccionamiento Estratégico: Proyección de la Innovación Tecnológica y Gestión Administrativa en las Pequeñas Empresas". Colombia. Recuperado el 02 de mayo de 2020 de https://scielo.conicyt.cl/scielo.php?script=sci_arttext\&pid=S0718-0764201700050000 3\&lng=es\&nrm=iso\&tlng=es

FRESCO, J.C. (2005). El proceso de transformación y cambio en las organizaciones. Argentina - Editorial IMR Consulting Group.

FRESCO J. C., MANUELIDES M., MARTÍNEZ D., ORTIZ S. y VILLEGAS R. (2015) "Cultura y cambio organizacional de las PyMES en la Ciudad de Rio Gallegos". Rio Gallegos, Santa Cruz, Argentina. Recuperado el 04 de Mayo de 2020 de https://dialnet.unirioja.es/servlet/articulo?codigo $=5278862$

LEY NACIONAL No 23.877 "Promoción y fomento de la innovación tecnológica" Buenos Aires, Argentina, 26 de octubre de 1990. Link: http://servicios.infoleg.gob.ar/infolegInternet/anexos/0-4999/277/norma.htm

LEY NACIONAL No 25.467 "CIENCIA, TECNOLOGIA E INNOVACION" Buenos Aires, Argentina, 20 de septiembre de 2001. Link: http://servicios.infoleg.gob.ar/ infolegInternet/anexos/65000-69999/69045/norma.htm

PERE ESCORSA C.- JAUME VALLS P. (2005) - Tecnología e Innovación de la Empresa. (2da ed.) Barcelona España - Ediciones UPC- Universitat Politécnica de Catalunya- 